Tecno Lógicas

ISSN 0123-7799

Vol. 17, No. 32, pp. 57-65

Enero-junio de 2014
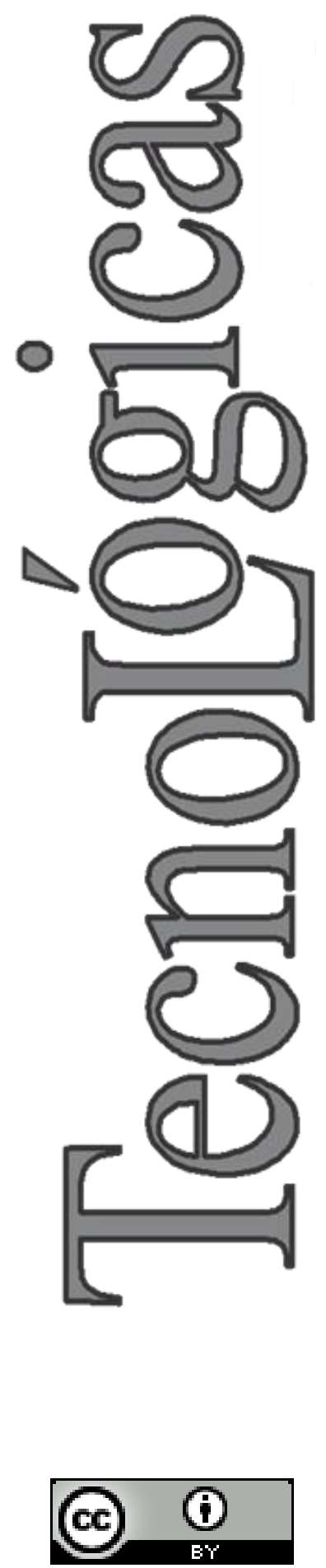

\section{Impacto de aguas grises en propiedades físicas del suelo}

\section{Grey water impact on soil physical properties}

\author{
Miguel L. Murcia-Sarmiento ${ }^{1}$, \\ Oscar G. Calderón-Montoya², Jaime E. Díaz-Ortiz ${ }^{3}$
}

Recibido: 16 de octubre de 2013, Aceptado: 09 de diciembre de 2013

Como citar / How to cite

M. L. Murcia-Sarmiento, O. G. Calderón-Montoya, J. E. Díaz-Ortiz, "Impacto de aguas grises en propiedades físicas del suelo", Tecno Lógicas, vol. 17, no. 32, pp. 57-65, 2014.

1 Ingeniero Agrícola, Ingeniero de recursos hídricos y cartografía, Corporación red país rural, Cali-Colombia, leomsart@gmail.com

2 Ingeniero Agrícola, Escuela Ingeniería de Recursos Naturales, Universidad del Valle, Cali-Colombia, osguicalmo@hotmail.com

3 Ingeniero Agrícola, Ph.D., Escuela Ingeniería de Recursos Naturales, Universidad del Valle, Cali-Colombia, jaime.diaz@correounivalle.edu.co 


\section{Resumen}

Debido a la creciente demanda de alimentos producida por el aumento de la población, el agua, como elemento indispensable en el ciclo del crecimiento de las plantas se convierte cada día en un aspecto fundamental de producción. La demanda por el uso de este recurso hace necesario la búsqueda de alternativas que deben ser evaluadas para evitar posibles impactos negativos. En este trabajo se evaluaron las modificaciones en algunas propiedades físicas del suelo regadas con aguas grises sintéticas. Se usó un diseño experimental de un factor: agua gris doméstica y dos tratamientos agua sin tratar (T1) y agua tratada (T2). Las variables de respuesta que se examinaron en el suelo fueron: conductividad eléctrica (CE), porcentaje de sodio intercambiable (PSI), diámetro ponderado medio (DPM) y retención de humedad del suelo (RHS). El agua se utilizó en riego localizado de alta frecuencia, monitoreado por tensiómetros para la producción de un cultivo de fríjol (Phaseolous vulgaris L). Como sistema de filtración se empleó una unidad compuesta por un filtro de arena (FLA) y un humedal artificial de flujo subsuperficial (HFSS). Los tratamientos presentaron diferencias significativas en el PSI y el RHS. El sistema FLA+HFSS es una alternativa para el tratamiento de agua grises debido a la mayor retención de sodio.

\section{Palabras clave}

Aguas grises, conductividad eléctrica, filtro de arena, irrigación, sodio intercambiable.

\section{Abstract}

Due to the increasing demand for food produced by the increase in population, water as an indispensable element in the growth cycle of plants every day becomes a fundamental aspect of production. The demand for the use of this resource is necessary to search for alternatives that should be evaluated to avoid potential negative impacts. In this paper, the changes in some physical properties of soil irrigated with synthetic gray water were evaluated. The experimental design involved: one factor: home water and two treatments; without treated water (T1) and treated water (T2). The variables to consider in the soil were: electrical conductivity (EC), exchangeable sodium percentage (ESP), average weighted diameter (MWD) and soil moisture retention (RHS). The water used in drip irrigation high frequency was monitored by tensiometer for producing a bean crop (Phaseolous vulgaris L). As filtration system used was employed a unit composed of a sand filter (FLA) and a subsurface flow wetland artificial (HFSS). The treatments showed significant differences in the PSI and the RHS. The FLA+HFSS system is an alternative to the gray water treatment due to increased sodium retention.

\section{Keywords}

Grey water, electrical conductivity, sand filter, irrigation, exchangeable sodium. 


\section{INTRODUCCIÓN}

Las aguas grises domésticas pueden constituir una fuente hídrica importante en la producción de alimentos a pequeña escala, su utilización para este fin a nivel mundial, es creciente en zonas de escases hídrica. El incremento, la concentración de la población y la variación climática, exige una gestión adecuada del recurso hídrico, afectado por el deterioro ambiental producido, entre otros factores, por el vertimiento de Aguas Residuales Domésticas (ARD). Se estima que en el año 2025, la escasez de agua causará anualmente pérdidas globales de 350 millones de toneladas de alimentos. Según [1], lo anterior significa que el agua será uno de los principales factores limitantes de la alimentación para el futuro, lo cual conduce a buscar soluciones que mitiguen los impactos.

Las Aguas Grises Domésticas (AGD) son aquellas que provienen de lavamanos, duchas, lavadoras, lavaplatos y lavaderos, representando una fracción de $75 \%$ en volumen de Aguas Residuales Domésticas (ARD). El AGD difiere del ARD por su bajo contenido de materia orgánica y menor concentración de materiales con potencial de impacto ambiental negativo tales como: sales, aceites, productos de síntesis química y contaminantes microbianos [2], compuestos que provienen de jabones y detergentes [3]. El AGD sin tratamiento ha sido utilizado en la irrigación a pequeña escala, presentando impactos negativos en las propiedades del suelo [4] asociados principalmente a la dispersión de agregados debido a la acumulación de sodio [5], y a la modificación de propiedades hidrodinámicas de los suelos ocasionada por la acumulación de surfactantes, [2], [6], [7].

El tratamiento de AGD se presenta como una alternativa para mitigar las alteraciones anteriormente nombradas, considerando las diferentes características físicas, ambientales y socio-económicas existentes en las pequeñas y medianas poblaciones, las cuales representan un reto a la hora de seleccionar y proyectar tecnologías sostenibles. Los sistemas naturales tales como filtración lenta en arena y humedales artificiales, son alternativas de bajo costo, fáciles de operar, eficientes y robustos en comparación con los sistemas de tratamiento convencional [8].

La investigación evaluó el impacto de la reutilización de aguas grises sintéticas en las propiedades físicas, químicas del suelo y la producción del cultivo. Se estudió el reuso de AGD sintética como fuente de riego en el cultivo del fríjol calima (Phaseolous vulgaris L), en condiciones controladas (invernadero). El tratamiento de las AGD combinó dos formas de filtración; un filtro lento en arena (FLA) y un humedal artificial de flujo sub-superficial (HFSS).

\section{METOdOLOGía}

El experimento se realizó en el laboratorio de suelos y agua (LASA) de la Universidad del Valle, ubicado a $983 \mathrm{msnm}$, (latitud de $3^{\circ} 22^{\prime} 21.47 " \mathrm{~N}$ y longitud de $76^{\circ} 31^{\prime} 48.82^{\prime \prime}$ ), en el municipio de Santiago de Cali. El clima ambiental es cálido y seco, y el edáfico corresponde a un régimen de humedad ústico con temperatura isohipertérmica.

Se empleó un diseño experimental unifactorial tomando como factor experimental (Agua Gris Doméstica) expuesta a dos tratamientos. Los tratamientos empleados fueron: $\mathrm{T} 1$ = Tratamiento 1 , AGD sin tratar; $\mathrm{y}$ T2 = Tratamiento 2, AGD tratada por filtración (FLA y HFSS). Por cada bloque (3) se emplearon 18 unidades experimentales (materas) distribuidas en forma aleatoria y equitativa, nueve por tratamiento para cada bloque.

Las AGD fueron elaboradas de forma sintética, similar al componente propuesto por [1]. La proporción de cada componente del AGD, fue cuidadosamente aplicada por medio de jeringas calibradas para cada unidad, con una aproximación de 0,001 g (ver Tabla 1). 
Tabla 1. Componente modificado utilizado para la fabricación de las aguas grises. Fuente: Autores

\begin{tabular}{lc}
\hline Componente & Cantidad \\
\hline Agua & $500 \mathrm{~L}$ \\
Detergente en polvo & $40 \mathrm{~g}$ \\
Champú & $50 \mathrm{~g}$ \\
Aceite vegetal & $7,5 \mathrm{~g}$ \\
\hline
\end{tabular}

Las variables de respuesta se presentan en la Tabla 2, indicando el método y la frecuencia de medición; la frecuencia de medición (M) se relaciona con los periodos del cultivo (antes de sembrar -M1- y después de la cosecha -M2).

\begin{tabular}{lcc}
\multicolumn{3}{c}{ Tabla 2. Variables de respuesta. Fuente: Autores } \\
\hline \multicolumn{1}{c}{ Variable } & $\begin{array}{c}\text { Frecuencia de } \\
\text { medición }\end{array}$ & Flor \\
\hline $\begin{array}{l}\text { Retención de humedad } \\
\text { del suelo (RHS) a 0,5 bar }\end{array}$ & M1 y M2 & $\begin{array}{c}\text { Pressure } \\
\text { Membrane } \\
\text { Aparatus }\end{array}$ \\
$\begin{array}{l}\text { Diámetro Ponderado } \\
\text { Medio (DPM) }\end{array}$ & M1 y M2 & Yoder \\
$\begin{array}{l}\text { Porcentaje de Sodio } \\
\text { Intercambiable (PSI) } \\
\begin{array}{l}\text { Conductividad eléctrica } \\
\text { (CE) }\end{array}\end{array}$ M1 y M2 & $\begin{array}{l}\text { Análisis } \\
\text { químico }\end{array}$ \\
Producción & M2 & Potenciómetro \\
\hline & & Balanza \\
\hline
\end{tabular}

El experimento se desarrolló en invernadero bajo condiciones controladas (precipitación), en un suelo clasificado taxonómicamente como Udertic Haplustoll, perteneciente a la consociación Arroyo (AY). Este suelo se caracteriza por poseer drenaje interno lento y externo muy lento. Sus horizontes diagnósticos son de carácter epipedón-mólico y epipedón-cámbico. La vegetación natural ha sido destruida, aunque a sus alrededores se encuentran reductos de un bosque natural de Bambusa vulgaris, del cual se tomaron las muestras. La Tabla 3 presenta una caracterización física del sustrato, que fue secado al ambiente durante 3 días y tamizado a $2 \mathrm{~mm}$, con el fin de garantizar la homogeneidad. Se utilizaron 54 materas con dimensiones de $20 \mathrm{~cm}$ de diámetro y altura, las cuales se llenaron individualmente con $8 \mathrm{~kg}$ de suelo.
Tabla 3. Caracterización del sustrato a la profundidad máxima de extracción. Fuente: Autores

\begin{tabular}{ll}
\hline Característica & Valor \\
\hline Densidad aparente & $1,36 \mathrm{gr} / \mathrm{cm}^{3}$ \\
Textura & Franco-Arcillosa \\
Color en seco & $10 \mathrm{YR} 4 / 3$ Marrón \\
Color en húmedo & $10 \mathrm{YR} \mathrm{3/4} \mathrm{Amarillo}$ \\
Tipo de estructura inicial & Granular \\
Clase de estructura & Media \\
Perfil analizado & $(0-15) \mathrm{cm}$ \\
\hline
\end{tabular}

Para determinar las condiciones iniciales del suelo se tomó una muestra de 2000 $\mathrm{g}$, de los cuales $1000 \mathrm{~g}$ se usaron para las determinaciones físicas en el Laboratorio LASA y $1000 \mathrm{~g}$ se enviaron al laboratorio de servicios analíticos del Centro Interamericano de Agricultura Tropical, para su análisis químico. Para la condición final, de cada bloque se escogieron al azar, dos muestras de $200 \mathrm{~g}$ por tratamiento en cada uno de los bloques. Las muestras se realizaron a $15 \mathrm{~cm}$ de la superficie considerando que esta profundidad representa la profundidad efectiva de raíces del cultivo propuesto Phaseolous vulgaris L [9]. La producción por unidad experimental se determinó por peso de los granos de fríjol.

El caudal de diseño del sistema de riego $(20 \mathrm{~L} / \mathrm{d})$ se obtuvo con base en las necesidades hídricas del cultivo para la zona. El humedal artificial (HFSS) se construyó siguiendo la metodología propuesta por [10], [11]. El filtro de arena (FLA) siguiendo las sugerencias propuestas por [12].

La humedad del suelo se controló con un tensiómetro (Irrometer ${ }^{\circledR}$ de $15 \mathrm{~cm}$ ) localizado en la profundidad efectiva de raíces. Para el riego se emplearon probetas, aplicando la lámina de agua hasta alcanzar una humedad cercana a capacidad de campo. En promedio se aplicó una lámina de 9,5 mm, cada 1,92 días, con 8,1 $\mathrm{L}$ por tratamiento, para un total de 40 riegos durante 76 días (ciclo del cultivo), utilizando $645,3 \mathrm{~L}$, para los dos tratamientos. La calidad del agua fue monitoreada cada 10 días desde la siembra del cultivo y 
clasificada mediante los criterios de [13], [14].

Se escogió como cultivo el fríjol Calima (Phaseolous vulgaris L) por ser una variedad regional cercana al sitio del experimento y considerada de alto consumo [15]. Las semillas fueron certificadas por el Instituto Colombiano Agropecuario (ICA), la siembra se realizó previamente con el suelo bajo condiciones de capacidad de campo, colocando dos semillas por unidad experimental. Se efectuó un monitoreo diario de la germinación y el brote durante cinco días, y posteriormente se eliminó una plántula por unidad experimental. Se resalta que el cultivo no recibió fertilización en ninguna etapa para minimizar la interferencia de los fertilizantes en las propiedades químicas del suelo.

Se empleó un análisis de varianza con covariable, para las variables del suelo (1) y un análisis sencillo, para la producción del cultivo (2). Se utilizó el programa Minitab 16 para el análisis de los datos.

$\mathrm{Y}_{(\mathrm{ij} \mathrm{final)}}=\mathrm{Y}_{(\mathrm{ij} \text { inicial })}+\mu+\mathrm{T}_{\mathrm{i}}+\mathrm{B}_{\mathrm{j}}+\mathrm{E}_{\mathrm{ij}}$

$\mathrm{Y}_{\text {(ij final) }}=\mu+\mathrm{T}_{\mathrm{i}}+\mathrm{B}_{\mathrm{j}}+\mathrm{E}_{\mathrm{ij}}$

Donde $Y_{\text {(ij final) }}=$ Variable de respuesta en $\mathrm{M} 2, \mathrm{Y}_{(\mathrm{ij} \text { inicial })}=$ Variable de respuesta en M1 (covariable), $\mu=$ Media poblacional, $\mathrm{T}_{\mathrm{i}}=$ Efecto del i-ésimo tratamiento ( $\mathrm{T} 1 \mathrm{y}$ T2) (i: 1-2), $B_{j}=$ Efecto del j-ésimo bloque $(\mathrm{j}: 1-3), \mathrm{E}_{\mathrm{ij}}=$ Error experimental asociado al i-ésimo tratamiento (T1 y T2) en el i-ésimo bloque.

\section{RESULTADOS Y DISCUSIÓN}

La Tabla 4, presenta los resultados de los parámetros de calidad de agua. Las normas sobre calidad de agua para riego según la FAO, indican que el agua empleada en ambos tratamientos no presenta restricción de uso con relación a la CE y para la Relación de Adsorción de Sodio (RAS) existe una restricción de uso moderada a leve, para su empleo en riego. Sin embargo, es necesario considerar que a largo plazo se pueden producir efectos adversos sobre la tasa de infiltración del suelo. Hay que resaltar que los valores de RAS para T1 y T2 presentaron diferencias entre sí. En el T2 estas diferencias se relacionan con la remoción de sodio y el incremento de calcio y magnesio migrado del recipiente que contenía el humedal artificial. De acuerdo con USDA, ambos tratamientos (T1 y T2) se clasificaron como C2$\mathrm{S} 1$, indicando un contenido de sodio bajo y salinidad media.

Tabla 4. Parámetros de calidad de agua para riego $(n=8)$.

\begin{tabular}{|c|c|c|c|}
\hline \multicolumn{4}{|c|}{$\mathrm{T} 1$} \\
\hline Parámetro & $\begin{array}{c}\text { Promedio } \\
\mathrm{mg} / \mathrm{L}\end{array}$ & $\begin{array}{l}\text { Rango } \\
\mathrm{mg} / \mathrm{L}\end{array}$ & $\begin{array}{c}\text { Desviación } \\
\text { estándar }\end{array}$ \\
\hline $\mathrm{DBO}_{5}$ & 16,75 & $5-31$ & 8,96 \\
\hline DQO & 63 & $32-175$ & 48,77 \\
\hline $\mathrm{N}-\mathrm{NO}_{3}$ & 0,09 & $0,05-0,130$ & 0,06 \\
\hline $\mathrm{PO}_{4}$ & 1,55 & $0,17-3,68$ & 1,29 \\
\hline $\mathrm{SO}_{4}$ & 57,23 & $36,75-35,48$ & 17,83 \\
\hline $\mathrm{Na}$ & 34,99 & $19,80-81,54$ & 19,46 \\
\hline $\mathrm{Ca}$ & 15,26 & $14,62-16,12$ & 0,69 \\
\hline $\mathrm{Mg}$ & 3,64 & $3,22-3,93$ & 0,257 \\
\hline $\mathrm{CE}$ & 179,49 & $128,21-243,59$ & 0,05 \\
\hline RAS & 2,07 & $1,21-4,92$ & 1,18 \\
\hline \multicolumn{4}{|c|}{$\mathrm{T} 2$} \\
\hline Parámetro & $\begin{array}{c}\text { Promedio } \\
\text { mg/L }\end{array}$ & $\begin{array}{c}\text { Rango } \\
\mathrm{mg} / \mathrm{L}\end{array}$ & $\begin{array}{c}\text { Desviación } \\
\text { estándar }\end{array}$ \\
\hline $\mathrm{DBO}_{5}$ & 2,5 & $1-10$ & 3,12 \\
\hline DQO & 10,88 & $3-25$ & 7,04 \\
\hline $\mathrm{N}-\mathrm{NO}_{3}$ & 0,01 & $0-0,01$ & \\
\hline $\mathrm{PO}_{4}$ & 0,19 & $0,04-0,42$ & 0,14 \\
\hline $\mathrm{SO}_{4}$ & 42,64 & $27,71-60,73$ & 11,96 \\
\hline $\mathrm{Na}$ & 31,07 & $27,42-46,13$ & 6,29 \\
\hline $\mathrm{Ca}$ & 52,86 & $38,00-63,22$ & 7,9 \\
\hline $\mathrm{Mg}$ & 6,35 & $0,43-1.00$ & 0,549 \\
\hline $\mathrm{CE}$ & 230,77 & $192,31-256,41$ & 0,04 \\
\hline RAS & 1,08 & $0,87-1,80$ & 0,3 \\
\hline
\end{tabular}

Se observó un aumento de conductividad eléctrica para T2 (FLA+HFSS), lo cual se asocia a la evaporación del agua en el humedal (HFSS), éstos resultados son similares a los obtenidos por [16], este comportamiento se ha presentado en general para la mayoría de los métodos de tratamiento natural. 
La Tabla 5, presenta los resultados obtenidos para la Conductividad Eléctrica del Suelo $\left(\mathrm{CE}_{\mathrm{s})}\right.$ determinada en los momentos de producción (M1 y M2). Los suelos sometidos a la irrigación con T1 y T2 estuvieron por debajo del intervalo de ligeramente salinos ( $\left.\mathrm{CE}_{\mathrm{s}} 2-4 \mathrm{dS} / \mathrm{m}\right)$. En la Fig. 1 se observa el comportamiento de CE para los suelos irrigados con $\mathrm{T} 1$ y $\mathrm{T} 2$ comparados con el valor de $\mathrm{CE}_{\mathrm{s}}$ inicial del suelo.

En la $\mathrm{CE}_{\mathrm{s}}$, No se presentaron diferencias significativas $(\mathrm{p}>0.05)$ entre $\mathrm{T} 1$ y T2, con lo cual se puede decir que ambos tratamientos afectaron de forma similar al suelo, lo cual concuerda con los criterios establecidos por FAO y USDA.

Tabla 5. Valores medios de $\mathrm{CE}_{\mathrm{s}}$, PSI, RHS, DPM y producción*, inicial y final, después de la irrigación con T1 y T2 $(n=3)$. Fuente: Autores

\begin{tabular}{lccc}
\hline Género & M1 & $\begin{array}{c}\text { M2 } \\
\text { T1 }\end{array}$ & T2 \\
\hline CE (dS/m) & 0,43 & 0,42 & 0,4 \\
PSI (\%) & 0,27 & 0,45 & 0,39 \\
RHS (\%) & 24,45 & 27,48 & 28,75 \\
DPM (mm) & 2,64 & 2,26 & 2,18 \\
Producción (Ton/ha) & & 1,16 & 1,09 \\
\hline
\end{tabular}

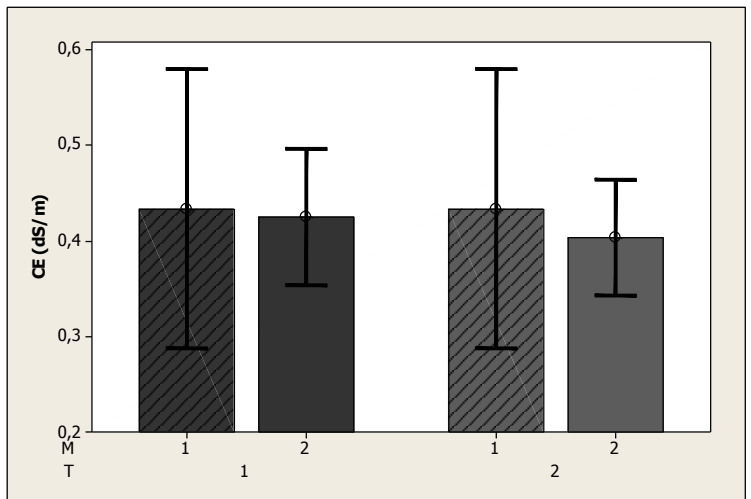

Fig. 1. Conductividad eléctrica del suelo. Fuente: Autores

La Tabla 5, presenta el resumen de los resultados de PSI para las condiciones M1 y M2, mostrando que independientemente del tratamiento empleado el suelo no adquirió a una condición sódica, lo cual concuerda con lo establecido por [17]. En esta medida se puede decir que el riego con $\mathrm{T} 1 \mathrm{y}$ T2 ligado al corto periodo del cultivo, no alcanzó a variar el PSI de manera signifi- cativa para superar el límite de "ligeramente sódico".

Sin embargo, los tratamientos T1 y T2 mostraron diferencias significativas de PSI entre los tratamientos T1 y T2 ( $\mathrm{p}=0,0375)$; como se ve en la Fig. 2. Esto puede atribuirse al sistema de filtración propuesto (FLA+HFSS) para el tratamiento T2 debido, el cual puede favorecer la extracción de sodio. Igualmente, la RAS de T1 $(2,072)$ también fue mayor a T2 (1,079). Aparentemente la adición de calcio y magnesio explica estos resultados, indicando que aunque la calidad del agua de los tratamientos fue clasificada de igual forma según FAO y USDA, los resultados indican que el tratamiento T2, tiene mayor efectividad en la disminución de acumulación del sodio intercambiable en el suelo.

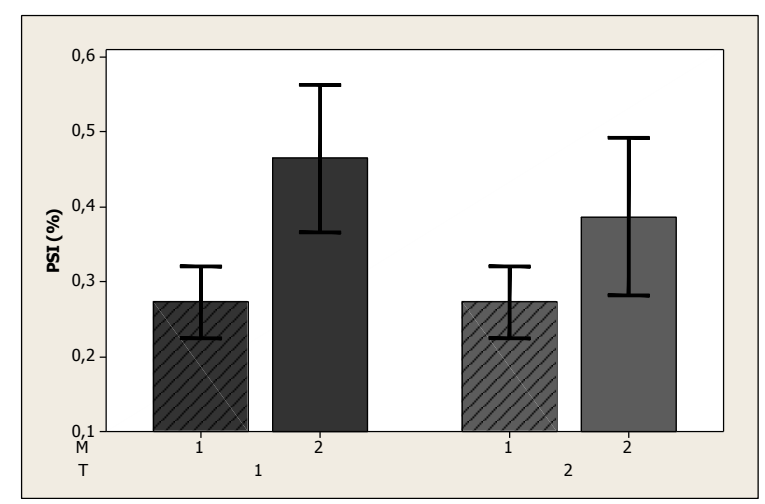

Fig. 2. Porcentaje de sodio intercambiable. Fuente: Autores

La Fig. 3, presenta los resultados de la retención de humedad del suelo (RHS) mostrando que es superior en el T2. El análisis de varianza realizado señala que existen diferencias significativas al nivel del $80 \%$ entre los tratamientos $(p=0,122)$. El aumento de la RHS se puede explicar por la presencia de Tensoactivos, componentes de los detergentes y jabones, en las AGD, los cuales reducen la tensión superficial del agua, permitiendo a su vez la fácil adsorción de esta por el suelo. En [18], aplicaron dos tensoactivos comerciales a suelos hidrófobos (Primer 604 y Aqueduct) en las dosis recomendadas por los fabricantes en diferentes localidades de Estados 
Unidos de América, Holanda, Australia y Alemania, encontrando en todos los casos reducciones del grado de repelencia.

El comportamiento de la RHS obtenida muestra concordancia con lo reportado por [2], [7] y [19] con relación a los efectos del AGD sobre las propiedades hidrodinámicas de los suelos. Es importante resaltar que estos pueden ser negativos o positivos dependiendo del comportamiento hidrófilo o hidrófobo del suelo, que condiciona la estructura bipolar de la molécula componente de los tensoactivos.

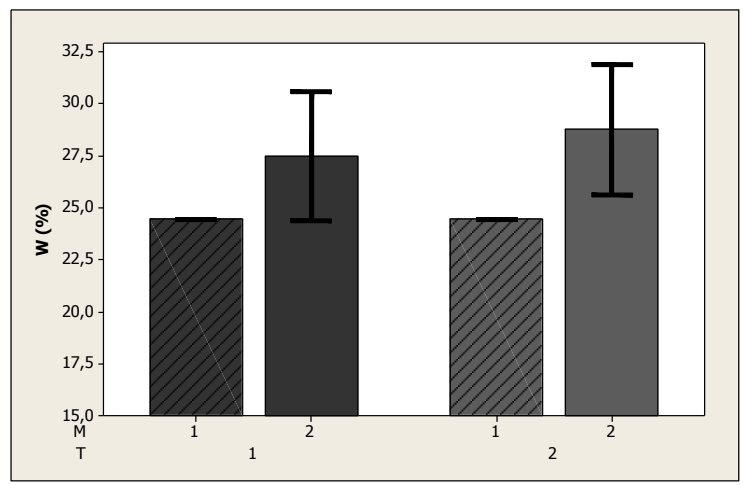

Fig. 3. Retención de humedad del suelo. Fuente: Autores

Los resultados de RHS (Tabla 5) señalan que el suelo se comportó moderadamente estable, para todos los tratamientos durante el tiempo del experimento. Con relación a la RHS el análisis estadístico entre los tratamientos no presentó diferencias significativas, resultado coherente con los criterios de la FAO, mostrando que ambos tratamientos, presentan el mismo riesgo de disminución de la tasa de infiltración (moderado a leve), propiedad que está fuertemente asociada a la estructura del suelo.

La Fig. 4, presenta la disminución del DPM asociada al riego con los tratamientos T1 y T2. El comportamiento anterior asociado al corto tiempo del cultivo permite deducir que posiblemente para un riego frecuente durante periodos largos, el DPM se puede ver fuertemente afectado, debido a la acumulación de Ion sodio en el suelo.

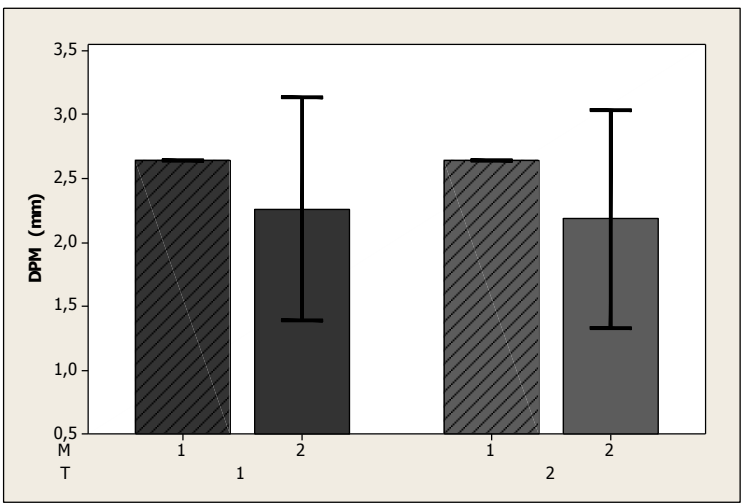

Fig. 4. Diámetro ponderado medio. Fuente: Autores

La Fig. 5, muestra los resultados de la producción del cultivo donde no se observan diferencias significativas entre la producción de las plantas para los dos tratamientos empleados. Aun así, la producción fue más alta para las plantas irrigadas con el T1, comportamiento que se asocia a la carga nutricional de este tratamiento. Las plantas irrigadas con ambos tratamientos estuvieron dentro del rango de producción de fríjol Calima según es reportado por [9]. Debido a que no se fertilizó el suelo durante el experimento todas las unidades experimentales presentaron clorosis; resultado que concuerda con lo reportado por [6].

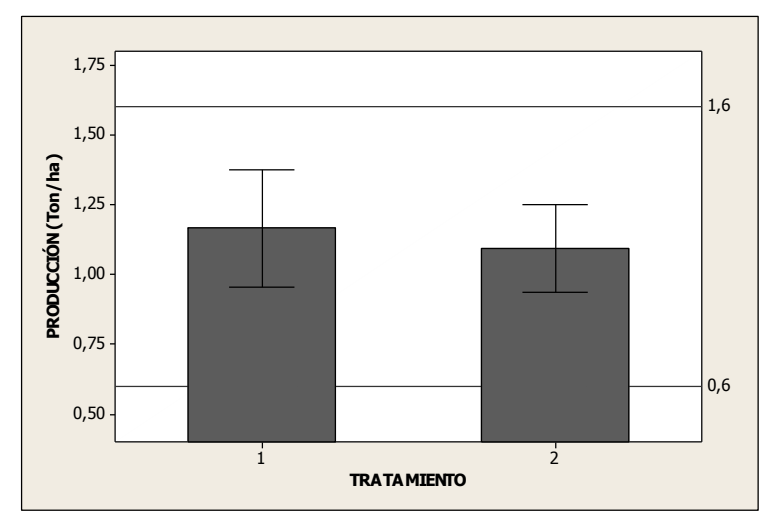

Fig. 5. Producción del cultivo. Fuente: Autores

\section{CONCLUSIONES}

El riego con aguas grises sintéticas sin tratar y aguas grises sintéticas tratadas mostró una tendencia al aumento de la 
retención de humedad del suelo, comportamiento que puede estar asociado a la carga de tensoactivos. Igualmente, se presentó un incremento en el contenido de sodio en el suelo. El empleo de aguas grises en irrigación prolongada exige tratamiento, debido a que se pueden presentar cambios negativos de la estructura del suelo, asociados a la acumulación de Ion Sodio en el complejo de cambio.

El sistema de tratamiento FLA+HFSS mostró ser un alternativa viable en el tratamiento de aguas grises sintéticas, gracias a la depuración de ion sodio, lo cual entregó un efluente con una RAS menos nociva, característica reflejada al comparar el porcentaje de sodio intercambiable de los suelos irrigados con aguas grises sintéticas y aguas grises sintéticas tratadas mediante el sistema FLA+HFSS. El riego con aguas grises sintéticas y aguas grises sintéticas tratadas no afecta de manera negativa la producción del cultivo, pero se nota una leve tendencia de incremento en la producción con las plantas regadas con agua gris sintética sin tratar, asociada a la carga nutricional presente, comportamiento que puede implicar en un corto plazo ahorro en fertilizantes, pero a largo plazo un deterioro en las condiciones físicas del suelo.

La implementación de las aguas grises sintéticas y aguas grises sintéticas tratadas como fuente de riego mostró que el reuso de aguas grises como fuente de riego se debe hacer teniendo en cuenta las características iníciales del suelo, asociando estas a la calidad del agua que se va a emplear, dado que con ello se pueden identificar los posibles impactos y las medidas de mitigación de los mismos, asociadas a la programación y aplicación correctas del riego.

\section{AGRADECIMIENTOS}

Este trabajo se realizó gracias al apoyo de la Vicerrectoría de Investigación de la Universidad del Valle.

\section{REFERENCIAS}

[1] J. C. Mira, "El agua en Colombia," 2006. [Online]. Available: http://www.ecoportal.net. [Accessed: 01Nov-2011].

[2] M. J. Travis, N. Weisbrod, and A. Gross, "Accumulation of oil and grease in soils irrigated with greywater and their potential role in soil water repellency.," Sci. Total Environ., vol. 394, no. 1, pp. 6874, May 2008.

[3] B. Jefferson, A. Laine, S. Parsons, T. Stephenson, and S. Judd, "Technologies for domestic wastewater recycling," Urban Water, vol. 1, no. 4, pp. 285-292, Dec. 2000.

[4] M. Abu-Zreig, R. P. Rudra, and W. T. Dickinson, "Effect of Application of Surfactants on Hydraulic Properties of Soils," Biosyst. Eng., vol. 84, no. 3, pp. 363-372, Mar. 2003.

[5] R. K. Misra and A. Sivongxay, "Reuse of laundry greywater as affected by its interaction with saturated soil," J. Hydrol., vol. 366, no. 1-4, pp. 55-61, Mar. 2009.

[6] A. Wiel-Shafran, Z. Ronen, N. Weisbrod, E. Adar, and A. Gross, "Potential changes in soil properties following irrigation with surfactant-rich greywater," Ecol. Eng., vol. 26, no. 4, pp. 348-354, Jul. 2006.

[7] M. Lado and M. Ben-Hur, "Treated domestic sewage irrigation effects on soil hydraulic properties in arid and semiarid zones: A review," Soil Tillage Res., vol. 106, no. 1, pp. 152-163, Dec. 2009.

[8] M. R. Peña, M. Van Ginneken, and C. Madera, "Humedales de flujo sub-superficial: Una alternativa natural para el tratamiento de aguas residuales domesticas en zonas tropicales," Rev. Ing. y Compet., vol. 5, no. 1, pp. 27-35, 2003.

[9] Centro Internacional de Agricultura Tropical, "Morfología de la planta de fríjol común (Phaseolous vulgaris L.)," Cali, 1984.

[10] US Environmental Protection Agency, "Guidelines for water reuse," Cincinnati, 1988.

[11] S. C. Reed, R. W. Crites, and E. J. Middlebrooks, Natural Systems for Waste Management and Treatment. McGraw-Hill, 1995, p. 433.

[12] J. T. Vissecher, R. Paramasivam, A. Raman, and H. A. Heijnen, Filtración lenta en arena tratamiento de agua para comunidades, 1era ed. CINARA-IRC, 1992, pp. 28-43.

[13] Food and Agriculture Organization of the United Nations, "Water Quality for Agriculture, Irrigation and Drainage," Rome, 1985.

[14] United States Department of Agriculture, Diagnosis and improvements of saline and alkali soils. Washington D.C.: USDA, 1954, pp. 128-129.

[15] J. Arias, T. Rengifo, and M. Jaramillo, "Buenas Prácticas Agrícolas en la Producción de Fríjol Voluble," Food and Agriculture Organization, 2007.

[16] C. Madera, J. P. Silva, and M. R. Peña, "Sistemas combinados para el tratamiento de aguas residuales basados en tanques sépticos filtro anaerobio y humedales sub-superficiales," Rev. Ing. y Compet., vol. 7, no. 2, pp. 5-10, 2005.

[17] F. Pizarro, Riegos localizados de alta frecuencia (RLAF): goteo, micro-aspersión, exudación, $2 \mathrm{da}$ ed. Madrid: Ediciones Mundi-Prensa, 1990, pp. 256-285. 
M. L. Murcia-Sarmiento et al. / Impacto de aguas grises en propiedades físicas del suelo

[18] S. Kostka, K. Dekker, C. J. Oostindie, C. M. Ritsema, D. Miller, and E. Karcher, "Advances in understanding and managing water repellent soils," 2011. [Online]. Available:

http://www.aquatrols.com/ResearchInfo/Understandin gWaterRepellency.html. [Accessed: 01-Apr-2011].
[19] M. J. Travis, A. Wiel-Shafran, N. Weisbrod, E. Adar, and A. Gross, "Greywater reuse for irrigation: effect on soil properties.," Sci. Total Environ., vol. 408, no. 12, pp. 2501-8, May 2010. 